\title{
Filter Design Problems with Convex Optimization
}

\author{
Sachin Rastogi \\ Department of Mathematics \\ Hindu College Moradabad \\ UP India
}

\author{
Sanjeev Rajan \\ Department of Mathematics \\ Hindu College Moradabad \\ UP India
}

\begin{abstract}
In this paper we consider the design of FIR filters that satisfy magnitude specifications. We refer to such design problems as magnitude filter design problems. In this paper it is shown that by a change of variables, a wide variety of magnitude filter design problems can be posed as convex optimization problems, i.e., problems in which the objective and constraint functions are convex.
\end{abstract}

\section{Keywords}

finite-duration impulse response (FIR), convex optimization, filter design, spectral factorization.

\section{INTRODUCTION}

A digital filter is a computational tool to extract useful information and remove undesired components from input sequences, and simultaneously generate output sequences.

In general, digital filters can be classified into two categories according to the

duration of their impulse responses, finite-duration impulse response (FIR) and infinite duration impulse response (IIR).

Generally, an FIR digital filter design can be equivalently formulated as a convex optimization problem in a finitedimensional linear space. Accordingly, its globally optimal solution can be achieved using various optimization techniques.

However, when magnitude and phase responses are both under consideration, in general, it is hard to transform an IIR filter design problem into an equivalent convex optimization problem. Hence, globally optimal solutions cannot be definitely attained

The mathematics of convex optimization has been studied for about one century. However, new research interests in this topic have been rejuvenated due to the advances of interiorpoint methods developed in the 1980s. Recently, many applications of convex optimization have been discovered in various fields of applied science and engineering, such as automatic control system, signal processing, VLSI circuit design, mechanical structure design, statistics and probability, and finance. There are many advantages of utilizing convex optimization to solve practical engineering problems. The most important one is that when a problem is equivalently cast as a convex optimization problem, any local solution is also a global optimum. Furthermore, a convex optimization problem can be solved very efficiently and reliably, using interiorpoint methods $[9,10]$.

It has been shown that given a desired frequency response, the WLS and minimax FIR filter design problems can be cast as equivalent convex optimization problems. Thus, the optimal designs can be definitely obtained.

A Finite impulse response (FIR) filter is a linear system described by a convolution input-output relation.

$$
Y(t)=\sum_{1=0}^{n-1} h(i) u(t-i)
$$

Where $\mathrm{u}: \mathbf{Z} \rightarrow \mathbf{R}$ is the input signal and $\mathrm{y}: \mathbf{Z} \rightarrow \mathbf{R}$ is the output signal. We say $\mathrm{n}$ is the filter order, and $h=(h(0), h(1), \ldots \ldots, h(n-1)) \in R^{n}$ are the filter coefficients. The filter frequency response $\mathrm{H}: \mathbf{R} \rightarrow \mathbf{C}$ is defined as

$$
H(\omega)=h(0) h(1) e^{-j \omega}+\ldots+h(n-1) e^{-j(n-1) \omega}
$$

Where $j=\sqrt{-1}$. Since $\mathrm{H}$ is $2 \pi$ periodic and satisfies $H(-\omega)=\overline{H(\omega)}$, magnitude specification has the form

$$
L(\omega) \leq|H(w)| \leq \text { for all } \omega \in[0, \pi]
$$

We refer to $\mathrm{L}$ and $\mathrm{U}$ as the (lower and upper) frequency response bound functions.

We can assume that $0 \leq L(\omega) \leq U(\omega)$ for all $\omega \in[0, \pi]$, since $L(\omega)$ can be replaced by $\max$ $\{L(\omega), 0\}$ without changing the constraint, and if $L(\omega)>U(\omega)$ for some $\omega$ then the magnitude specification is evidently infeasible.

The design problem can be expressed as the optimization problem.

$$
\begin{aligned}
& \text { Minimize } \delta \\
& \text { Subject to } 1 / \alpha|H(\omega)| \leq \alpha, \omega \in\left[0, \omega_{p}\right] \\
& |H(\omega)| \leq \delta, \omega \in\left[\omega_{s}, \pi\right] .
\end{aligned}
$$

The optimization variables are the filter coefficients $h \in R^{n}$ and the stop-band attenuation (bound) $\delta \in R$. The Problem parameters are the filter order $\mathrm{n}$, the passband frequency $\omega_{p}$, the stop band frequency $\omega_{s}$, and the maximum allowed passband ripple.

In this paper we extend the idea and apply nonlinear convex Optimization techniques to a variety of magnitude 
filter design problems. Linear and quadratic programming are well-developed fields; extremely efficient software is widely available. Making use of convex optimization methods more general than linear or quadratic programming preserves the solution efficiency, and allows us to handle a wider class of problems, e.g. problems with logarithmic (decibel) objectives.

\section{SPECTRAL FACTORIZATION}

The autocorrelation coefficients associated with the filter (1.1) are defined as

$$
r(t)=\sum_{I=-n+1}^{n-1} h(i) h(i+t), t \in Z,
$$

where we interpret $\mathrm{h}(\mathrm{t})$ as zero for $t<0$ or $t>n-1$, Since $r(t)=r(-t)$ and $r(t)=0$ for $t \geq n$, it suffices to specify the autocorrelation coefficients for $t=0, \ldots \ldots . n-1$, with some abuse of notation, we will write the autocorrelation coefficients as a vector $r=(r(0), \ldots . r(n-1)) \in R^{n}$

The Fourier transform of the autocorrelation coefficients is

$$
R(\omega)=\sum_{t \in Z} n(t) e^{-j \omega t}=r(0)+\sum_{t=1}^{n-1} 2 r(t) \cos \omega t=\left.H(\omega)\right|^{2}
$$

i.e., the squared magnitude of the filter frequency response. We will use the autocorrelation coefficients $r \in R^{n}$ as the optimization variable in place of filter coefficients $h \in R^{n}$. This change of variables has to be handled carefully, since the transformation from filter coefficients into autocorrelation coefficients is not One to one, and not all vector $r \in R^{n}$ are the autocorrelation coefficients of some filter.

The spectral factorization theorem states that are there exists an $h \in R^{n}$ such that $r \in R^{n}$ is the autocorrelation coefficients of $h$ if and only if.

$$
R(\omega) \geq 0 \text { forall } \omega \in[0, \pi] .
$$

The process of determining filter coefficients $\mathrm{h}$ whose autocorrelation coefficients are $\mathrm{r}$, given an $r \in R^{n}$ that satisfies the spectral factorization condition (1.7), is called spectral factorization.

The magnitude specification (1.3) can be expressed in terms of the autocorrelation coefficients $r$ as

$$
L(\omega)^{2} \leq R(\omega) \leq U(\omega)^{2} \text { for all } \omega \in[0, \pi] .
$$

If we consider $\mathrm{r}$ that are the autocorrelation coefficients of some h,

$$
L(\omega)^{2} \leq R(\omega) \leq U(\omega)^{2}, R(\omega) \geq 0 \text { for all } \omega \varepsilon[0, \pi] .
$$

These conditions are equivalent to the original magnitude specification in the following sense: there exists an $h$ that satisfies (1.3) if and only if there exists an $r$ that satisfies (1.8). Note that the spectral factorization constraint
$R(\omega) \geq 0$ is redundant, it is implied by

$$
L(\omega)^{2} \leq R(\omega) .
$$

For each $\omega$. the constraints in (1.8) are a pair of linear inequalities in the vector $r$; hence the overall constraint (1.8) is conver in $r$

\section{CONVEX OPTIMIZATION}

The convex optimization problems of the general form

$$
\begin{aligned}
& \text { Minimize } f_{0}(x) \\
& \text { Subject to } A x=b, \\
& \qquad f_{i}(x) \leq 0, i=1 \ldots \ldots ., m,
\end{aligned}
$$

$g_{i}(x, \omega) \leq 0, \omega \in[0, \pi], i=1, \ldots \ldots \ldots \ldots \ldots, p$,

where $x \omega \in R^{k}$ is the optimization variable, $\mathrm{f}_{0}, \ldots \ldots, \mathrm{f}_{\mathrm{m}}$ : $\mathrm{R}^{\mathrm{k}} \rightarrow \mathrm{R}$ are convex functions, and for each $\omega \in[0, \pi], g_{i}(x, \omega)$ are convex functions of $x$. Note the three types of constraints: $A x=b$ are the equality constraints; $f_{i}(x) \leq 0$ are the (ordinary) inequality constraints; and $g_{i}(r, \omega) \leq 0$ for all $\omega$ are the semiinfinite inequality constraints. If the objective function $f_{0}$ is identicall zero, the problem (1.10) reduces to verifying whether the constraints are feasible or not, i,e., to a feasibility problem.

The semi-infinite inequality constraint

$$
g_{i}(x, \omega) \leq 0, \text { for all } \omega \in[0, \pi],
$$

can be handled by expressing it as the ordinary inequality constraint

$$
\begin{gathered}
h_{i}(x)=\sup g_{i}(x, \omega) \leq 0 . \\
\omega \in[0, \pi]
\end{gathered}
$$

It is easily verified that $\mathrm{h}_{\mathrm{i}}$ is a convex function of $x$, since for each $\omega, g_{i}(x, \omega)$ is convex in $\omega$. (On the other hand, $h_{i}$ is often nondifferentiable, even if the function $\mathrm{g}_{\mathrm{i}}$ are differentiable.) Thus, the semi-infinite constraints in (1.10) can be handled by several methods for general (nondifferentiable) convex optimization.

The semi-infinite constraints can also be approximated in a very straight forward way by sampling or discretizing frequency. We choose a set of frequencies.

$$
0 \leq \omega_{i} \leq \omega_{2} \leq \ldots \ldots \ldots \ldots . \ldots \omega N \leq \pi,
$$

oftern uniformly or logarithmically spaced, and replace the semi-indifinite inequality constraint.

$$
g_{i}(x, \omega) \leq 0, \text { for all } \omega \in[0, \pi],
$$

with the set of $\mathrm{N}$ ordinary inequality constraints 


$$
g_{i}\left(x, \omega_{k}\right) \leq 0, K=1, \ldots \ldots \ldots \ldots, N .
$$

When $\mathrm{N}$ is sufficiently large, discretization yield a good approximation of the SIP [19].

As an example consider the lowpass filter design problem given by (1.9).

The discretized approximation has the form.

$$
\operatorname{Max} \bar{\delta}
$$

Subject to $\quad 1 / \alpha^{2} \leq R\left(\omega_{k}\right) \leq \alpha^{2}, \quad \omega_{k} \in\left[0, \omega_{p}\right]$

$$
\begin{aligned}
& R\left(\omega_{k}\right) \leq \hat{\delta}, \quad \omega_{k} \in\left[\omega_{s}, \pi\right] \\
& R\left(\omega_{k}\right) \geq 0, \quad \omega_{k} \in[0, \pi]
\end{aligned}
$$

This is in fact a linear program (LP) with $\mathrm{n}+1$ variables ( $\mathrm{r}, \square$ ), and $2 \mathrm{~N}$ linear inequality constraints.

The sampled version of the problem is an outer approximation of the original problem, its feasible set includes the feasible set of the original problem. In many cases this causes no particular harm, especially if $\mathrm{N}$ is large. But if the spectral factorization condition does not hold. i.e., $R(v)<0$ for some $\square$ between samples, then spectral factorization breaks down; we can not find a set of filter coefficients $h$ that have $r$ as its autocorrelation. Several methods can be used to avoid this pitfall. The simplest is to add a small safety margin to the sampled version of the spectral factorization condition, i.e., replace it by

$$
R\left(\omega_{k}\right) \geq \varepsilon, K=1, \ldots \ldots \ldots N
$$

Where $\varepsilon$ is small and positive. This can be done in an ad hoc way, by increasing $\varepsilon$ (and re-solving the problem ) until the spectral factorization of $\mathrm{R}$ is successful. If $\mathrm{N}$ is large this will occur when $\varepsilon$ is small.

We can also analyze the approximation error induced by discretization by bounding the variation of the functions $g_{i}(x, \omega)$ for $\square$ between samples. To give a very simple example, assume we use uniform frequency sampling i.e., $\omega_{k}=(k-1 / 2) \pi / n, k=1, \ldots \ldots \ldots . . N . \quad$ we assume we have (or impose) some bound on the size of h, say,

$$
\|h\|=\sqrt{h(0)^{2}+\ldots \ldots \ldots+h(n-1)_{2} \leq M .} .
$$

Thus, $r(0)=\|h\|^{2} \leq m^{2}$, and a standard result show that $|r(t) \leq| M^{2}$ for all t.

Now let $\mathrm{w}$ be any frequency in $[0, \Pi]$ and denote the nearest sampling frequency, so that, $\left|\omega-\omega_{k}\right| \leq \pi(2 N)$. we have

$\leq 4 M^{2} \sum_{t=1}^{n-1}|\sin | \omega-\omega_{k}|t| \leq 4 M^{2} \sum_{\substack{t=1 \\|t=1|}}^{n-1}\left|\omega-\omega_{k}\right| t \leq M^{2} n(n-1) \pi / N$ Thus, the inters ample error cannot exceed $M^{2} n(n-1) \pi / N$ (which evidently converges to zero as $\mathrm{N} \rightarrow \infty)$. For example, if we take $\varepsilon M^{2} n(n-1) I I / N$ in (1.12), then it is guaranteed that the (semi-infinite) spectral factorization condition (1.7) will be met. The bound developed here is very simple, and only meant to give the general idea; far more sophisticated bounds can be derived.

In the remainder of this chapter we will pose problems first, as convex, semi-infinite problems. We then either give the sampled version, or point out if it has a special form such as LP. The sampled versions can be thought of as approximation (which are probably more than adequate for practical design) or as subproblems that arise in sophisticated algorithms that handle the semi-infinite constraints exactly.

\section{LOWPASS FILTER DESIGN}

In this section we consider the lowpass filter design problems. The same techniques are readily applied to other filters such as highpass, bandpass, notch, bandstop, or complex filter types with multiple stop and pass bands. specification,

The constraints consist of a passband ripple

$$
1 / \alpha \leq|H(\omega)| \alpha \leq \mid \text { for } \omega \in\left[0, \omega_{p}\right],
$$

where $\omega_{p}$ is the passband frequency, and $\alpha \geq 1$ gives the passband ripple. The stopband attenuation specification is given by

$|H(\omega)| \leq \delta$ for $\omega \in\left[\omega_{s,} \pi\right]$,

where $\omega_{p}$, is the stopband frequency, and $\delta$ gives the maximum stopband gain. These specification are illustrated in figure 1 .

We have seen that the problem of maximizing stopband attenution (i.e., minimizing $\delta$ ) can be formulated as convex optimization problem.

$$
\text { Minimize } \bar{\delta}
$$

Subject to $1 / \alpha^{2} \leq R(\omega) \mid \alpha^{2} \omega \in\left[0, \omega_{p}\right]$,

$$
R(\omega) \geq 0, \omega \in[0, \pi],
$$

which, when discretized in frequency, yields an LP.

We can consider several variations on this problem. Suppose we fix the stopband attenuation and wish to minimize the passband ripple. This can be expressed as the optimization problem.

$$
\text { Minimize } \bar{\alpha}
$$

$$
\begin{array}{r}
\text { Subject to } 1 / \bar{\alpha} \leq R(\omega) \mid \bar{\alpha} \omega \in\left[0, \omega_{p}\right], \\
R(\omega) \geq 0, \omega \in[0, \pi],
\end{array}
$$

where the optimization variables are $\mathrm{r}$ and $\bar{\alpha}$. The problem parameters are $\delta, \omega_{p}, \omega_{s}$ (and the filter order $\mathrm{n}$ ). (The optimization variable $\bar{\alpha}$ corresponds to $\alpha^{2}$ in the ripple specification.)

This problem is in fact a conver optimization problem. To see this we consider the specifications at a fixed frequency $\mathrm{w}$. the constraints 


$$
R(\omega) \leq \bar{\alpha}, R(\omega) \leq \delta^{2}, \quad(\omega) \geq 0
$$

Are linear inequalities on the variables $(\mathrm{r}, \alpha)$. The remaining, nonlinear constraint is

$$
1 / \bar{\alpha}-R(\omega) \leq 0
$$

The function $1 / \bar{\alpha}-R(\omega)$ can be verified to be conver in the variables (r, $\bar{\alpha}$ ) (since $\bar{\alpha}>0$ ). Indeed, when sampled this problem can be very efficiently solved as a second-order come program (SOCP). The passband ripple minimization (in $\mathrm{dB}$ ) cannot be solved (directly) by linear programming; it can, however, be solved by nonlinear convex optimization.

It is also possible to include several types of constraints on the slop of the magnitude of the frequency response. We start by considering upper and lower bounds on the absolute slop, i.e.,

$$
a \leq d / h(\omega) \mid \leq b .
$$

This can expressed as

$$
a \leq \frac{d R(\omega)^{1 / 2}}{d \omega}=\frac{\frac{d R}{d \omega}}{2 \sqrt{R \omega}} \leq b
$$

which (since $R(\omega)$ is constrained to be positive) we can rewrite as

$$
2 \sqrt{R(\omega)} a \leq d R / d \omega \leq 2 \sqrt{R(\omega)} b .
$$

Now we introduce than assumption that $a \leq 0$ and $b \geq 0$. The inequalities can be written.

$$
2 a \sqrt{R(\omega)-d R / d \omega} \leq 0, \frac{d R}{d \omega}-b \sqrt{R(\omega)} \leq 0 .
$$

Since $R(\omega)$ is a linear function of $\mathrm{r}$ (and positive), $\sqrt{R(\omega)}$ is a concave function or $\mathrm{r}$. Hence the function $a \sqrt{R(\omega)}$ and $-b \sqrt{R(\omega)}$ are convex (since $a \leq 0$ and $b \geq 0$.) Thus, the inequalities (1.13) are convex (since $\frac{d R}{d \omega}$ is a linear function of $\mathrm{r}$ ).

\section{LOGARITHMIC APPROXIMATION}

Consider the problem of designing an FIR filter so that its frequency response magnitude best approximates a target or desired function, in the sense of minimizing the maximum approximation error in decibels $(\mathrm{dB})$.

We can formulate this problem as

$$
\sup _{\omega \in[0, \pi]} \log |H(\omega)|-\log D(\omega) \mid
$$

where $D:[0, \pi] \rightarrow R$ is the desired frequency response magnitude (with $D(\omega) a \leq 0$ for all $\omega$ ). We call (1.14) a logarithmic chebychev approximation problem, since it is a minimax (Chebychev) Problem on a logarithmic scale.

as

We can express the log-Chebychev problem (1.14)

Minimize $a$

Subject to $1 / a \leq R(\omega) / D(\omega)^{2}<a, \omega \in[0, \pi]$,

$$
R(\omega) a \leq 0, \omega \in[0, \pi]
$$

where the variable are $r \in R^{2}$ and $a \in R$ This is a convex optimization problem. Simple variation on this problem includes the addition of other constraints or a frequencyweighted log-Chebychev objective.

\section{MAGNITUDE EQUALIZER DESIGN}

In the simplest magnitude equalizer problem, we are given a function $T:[0, \pi] \rightarrow C$ not necessarily the frequency response of an FIR filter), and need to design an FIR filter (equalizer) $\mathrm{H}$ so that the product $\mathrm{TH}$ has approximately constant magnitude, e.g., one;

$$
\text { | } T(\omega) H(\omega) \mid \approx 1 \text { for all } \omega \in[0, \pi] .
$$

Where the equalizer processes the signals before the given function; but the problem is the same if the order of the equalizer and give function, but the problem is the same if the order of the equalizer and given function is reversed. Not also that we only need to know the Magnitude $|T(\omega)|$, and not $T(\omega)$, for $\omega \in[0, \pi]$.

The equalizer problem can be posted as the logChebychev approximation problem.

$$
\sup _{\omega \in[0, \pi]}\|\log |T(\omega)| H(\omega)\|
$$

which is readily formulated as a convex problem using the autocorrelation coefficients $r$ as the design variables:

$$
\begin{array}{cc}
\text { Minimize } & \alpha \\
\text { Subject to } & 1 / \alpha \leq R(\omega) / T(\omega)^{2}<a, \omega \in[0, \pi] \\
R(\omega) \leq 0, \omega \in[0, \pi]
\end{array}
$$

In many applications we must add regularization constraints on the equalizer frequency response, to keep the magnitude or its slope from being too large. These constraints are readily handled. For example, we can impose (frequencydependent) bounds on $|\mathrm{H}|$ and its absolute or logarithmic derivative, as described above. A very simple way to bound the size of $h$ is to impose the constraint.

$$
r(0)=h(0)^{2}+\ldots \ldots+h(n-1)^{2} \leq M^{2}
$$

(which is a single linear inequality on $r$ ).

So far we have assumed that the target equalized gain, i.e., the desired valus of $\mid \mathrm{TH}$, is one. We can also allow some freedom in the target value of the equalized gain. This type of problem can be handled using an absolute (or more accurately, squared) criterion. 


$$
\sup _{\omega i n i m i z e} \mid T(\omega) H(\omega)^{2}=\gamma
$$

where $\gamma$ is subject to some bounds such as $\gamma_{1} \leq \gamma_{2} \leq \gamma_{h}$, and $h$ is subject to some regularization constraints. Note that the optimization variables here are $h$ and $\gamma$.

we can cast this as the convex problem

$$
\begin{aligned}
& \text { Minimize } \\
& \text { Subject to } \\
& |R(\omega)| T(\omega)^{2}-\gamma \mid \leq \alpha, \omega \in[0, \pi], \\
& \qquad R(\omega) \geq 0, \omega \in[0, \pi]
\end{aligned}
$$

where in addition we have limits on $\gamma$ and some construction on $\mathrm{H}$ ( i.e.., R). If this problem is discretized, it becomes an LP.

We can formulate the multi system magnitude equalization problem as a minimax log - Chebychev approximation problem :

$$
\text { Minimize } \max \quad \max _{k=1, \ldots \ldots \ldots \in[0, \pi]} \sup _{\omega \in[}|\log | T_{K}(\omega) H(\omega) \|
$$

In this formulation we have fixed the target value for each |

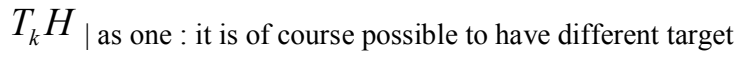
values for different $\mathrm{k}$. We should point out that this minimax formulation already builds in a form of regularization:

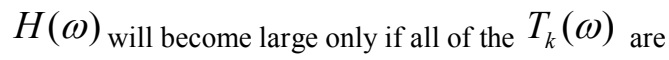
small.

The goal is to choose $\mathrm{H}$ so that the magnitude response at $y_{1} \ldots \ldots, y_{k}$ are all approximately over $\omega$.

We can formulate a minimax squared magnitude problem such as

$$
\left.\max _{k=1,2, \ldots k} \sup _{\omega \in[0, \pi]}\left\|T_{k}(\omega)\right\| H(\omega)\right|^{2}-\gamma k,
$$

To which we might add constraints on $\gamma_{k}$ such as a lower and upper bound. Here the variables here are $\mathrm{H}$ and $\gamma_{1}, \ldots \ldots . ., \quad \gamma_{k}$. This problem can be cast as the convex problem.

$$
\begin{gathered}
\text { Minimize } \alpha \\
\quad \text { Subject to } \\
\|\left. T_{k}(\omega)\right|^{2} R(\omega)-\gamma_{k} \mid \leq \alpha, k=1, \ldots . K, \omega \in[0, \pi]
\end{gathered}
$$$$
\text { This becomes an LP when discretized. }
$$

$$
\left[\Omega_{1}, \Omega_{2}\right],\left[\Omega_{2}, \Omega_{3}\right] \ldots \ldots . .\left[\Omega_{k}, \Omega_{k+1}\right]
$$

where $0<\ldots .<\Omega_{k+1} \leq \pi$. A common choice of frequencies differ by one-third octave, i..e .., $\Omega_{k}=\frac{2^{k-1}}{3} \Omega$ for $k=1, \ldots \ldots \ldots, \mathrm{K}$. The average gain of a function $\mathrm{G}$ : $[0, \pi]$ $\square$ C over the kth band $\left[\Omega_{\mathrm{k}}, \Omega_{\mathrm{k}+1]}\right.$, is defined by

$$
\left(\frac{1}{\Omega_{k+1}-\Omega_{k}} \int_{\Omega_{k}}^{\Omega_{k+1}}\left|G(\omega)^{2}\right| d \omega\right)^{1 / 2}
$$

Using a log- Chebychev (criterion for the gains and $r$ as the variable, we can express this equalization problem as

$$
\begin{gathered}
\text { Minimize } \alpha \\
\text { Subject to } \\
\frac{1}{\alpha} \leq \frac{1}{\Omega_{k+1}-\Omega_{k}} \int_{\Omega_{k}}^{\Omega_{k+1}} \quad k=1, \ldots \ldots, k \\
R(\omega) \geq 0 \quad \omega \in[0, \pi]
\end{gathered}
$$

This is a convex problem in $r$ and $\alpha$. To solve it numerically, we can approximate the integral by frequency sampling. (Indeed, $|\mathrm{T}|$ is likely to be given by its values at a fine sampling of frequencies, and not in some analytical form). We can also, of course add constraints on $\mathrm{H}$.

\section{LINEAR ANTENNA ARRAY WEIGH DESIGN}

Consider a liner array of $\mathrm{N}$ isotropic antennas spaced uniformly a distance $d$ apart in a plane as shown in figure 9. A plane harmonic wave of wavelength $\lambda$ is incident on the array from angle $\theta$. The antennas sample the incident wave, and the resulting singals are demodulated and then linearly combined with the antenna weights $\omega_{1} \ldots \ldots . ., \omega_{n} \in C_{\text {(which are our }}$ design variables) to form the combined out put of the antenna array, which is a complex number G. The array output G depends on the incidence angle $\theta$ of the incoming wave (and also the weight). As a function of the incidence angle $\mathrm{G}:[0, \pi]$ $\square \mathrm{C}$ is called the pattern function, and is given by

$$
G(\theta)=\sum_{k=0}^{n-1} \omega_{k} e^{-j k \Omega}
$$

where $\Omega$ depends on the incidence angle as

$$
\Omega=-\frac{2 \pi d}{\lambda} \cos \theta
$$

If we define $H:[-\pi \pi]$ as

$$
H(\Omega)=\sum_{k=0}^{n-1} \omega_{k} e^{-j k \Omega},
$$

Then we have $G(\theta)=H(\Omega)$. H is then the frequency response of an FIR filter with (complex) coefficients $\omega_{1}, \ldots \ldots \omega_{n}$.Since $\mathrm{H}$ does not satisfy $H(-\Omega)=H(\Omega)$ (as the frequency response of an FIR filter with real coefficients does), we specify $H$ over $\Omega \in[-\pi, \pi]$.

For $\theta \in[0, \pi] \Omega$ is monotonically increasing function of $\theta$, which we will denote $\Psi$,ï.e.., $\Psi(\theta)=-2 \pi \mathrm{d} / \lambda \cos \theta$. As the incidence angle $\theta$ varies from 0 to $\pi$, the variable, $\Omega=\psi(\theta)$ varies over the range $\pm 2 \pi_{\theta} / \lambda$. To simplify the discussion below, we make the (common) assumption that $d<\lambda / 2$, i.e.., the element spacing is less than one half wavelength. This 
implies that for $\theta \in[0, \pi], \varphi(\theta)=\Omega$ is restricted to an interval inside $[-\pi, \pi]$. An interval.

$$
\theta \min \leq \theta \leq \theta \max
$$

where $\theta \min , \theta \max \in[0, \pi]$, transforms under (1.18) to the

$\Omega \min \leq \Omega \leq \Omega \max$,

where $\Omega_{\min ,=\psi}(\theta \min )$ and $\Omega \max =\psi(\theta \max )$, which lie in $[-\pi, \pi]$.

By analogy with the FIR filter design problem, we can define an antenna pattern magnitude specification as $L(\theta) \leq|G(\theta)| \leq U(\theta)$ for all $\theta \in[0, \pi]$.

An antenna array weight design problem involves such specification. As a simple example suppose, we want the array to have approximately uniform sensitivity corresponding interval

for $\theta \in\left[0, \theta_{b}\right]$, and sensitivity as small as possible in the interval $\left[\theta_{\mathrm{s}}, \pi\right]$. This problem can be possible as

$$
\begin{gathered}
\text { Minimize } \gamma \\
\text { Subject to } 1 / \alpha \leq|G(\theta)| \leq \alpha, \quad \theta \in\left[0, \theta_{b}\right] \\
|G(\theta)| \leq \alpha, \quad \theta \in\left[\theta_{s}, \pi\right] .
\end{gathered}
$$

This problem is the analog of the lowpass filter design problem (1.4) Here

$\theta_{\mathrm{b}}$ denotes the ( half) beamwidth, $\theta_{\mathrm{s}}$ denotes the beginning of the side lobe, and is called the side lobe attenuation level.

$$
\text { Minimize } \delta
$$

Subject to

$$
\begin{aligned}
& 1 / \alpha \leq|H(\Omega)| \leq \alpha, \Omega \in\left[\psi(0), \psi\left(\theta_{b}\right)\right] \\
& |H(\Omega)| \leq \delta, \Omega \in\left[\psi\left(\theta_{s}\right), \psi(\pi)\right]
\end{aligned}
$$

Now (1.21) is a lowpass filter design problem, but with complex coefficients $\mathrm{w}_{\mathrm{i}}$ and specification over the interval [ $\pi, \pi]$. It can be handled like an FIR filter magnitude design problem, by an extension of spectral factorization to the complex case.

We define the (now complex) autocorrelation coefficients $\mathrm{r}(\mathrm{k})$, associated with $\omega$, as

$$
r(k)=\sum_{i=1}^{n-1} \omega_{i} \cdot \bar{\omega}_{l+k} \quad k=0, \ldots . n-1 .
$$

The Fourier transform of $r$ is

$$
R(\theta)=\sum_{k=-n-1}^{n-1} r(k) e^{-j k} \Omega=\left|G(\theta)^{2}\right|,
$$

is the squared magnitude of the antenna pattern function ( where $\theta$ and $\Omega$ is related as in (1.17)). We can use $r \in \mathrm{C}^{\mathrm{n}}$ as the design variables, provided we add the spectral factorization condition $\mathrm{R}(\theta) \geq_{0}$ for all $\theta \in[0, \pi]$.

The magnitude constraint can be expressed in terms of $\mathrm{R}$ as

$L(\theta)^{2} \leq R(\theta)^{2} \leq U(\theta)^{2}$ for all $\theta \in[0, \pi]$,

i.e., as an (infinite) set of linear inequalities on $\gamma$.

\section{CONCLUSIONS}

We have shown that a variety of magnitude FIR filter design problem can be formulated, in terms of the autocorrelation coefficients, as possibly nonlinear) convex semi-infinite optimization problems. As a result, the globally optimal solution can be efficiently computed. By considering nonlinear convex optimization problems, we can solve a number of problems of practical interest, e.g. minima decibel problems, with an efficiency not much less than standard methods that really on, for example, linear or quadratic programming.

\section{REFERENCES}

[1] F.A.Aliyev, B.A. Bordyug, and V.B. Larin Factorization of polynomial matrices and separation of rational matrries. Soviet Journal of Computer and Systems Sciences, 28(6):47-58,1990.

[2] F.A. Aliyev, B.A. Bordyug, and V.B. Larin Discreate generalized algebraic Riccati equations and polynomial matrix factorization. Syst. Control Letters, 18:4959,1992 .

[3] B.Anderson. An algebraic solution to the spectral factorization problem. IEEE Trans. Aut. Contral, Ac12(4):410-414, Aug.1967.

[4] B.Anderson. Anderson and J.B. Moore. Optimal Filtering. Prentice-Hall, 1979.

[5] B.Anderson and S.Vongpanitlerd. Network analysis and synthesis modern system theory approch. PreticeHall,1973.

[6] B.D.O. Anderson and K.L.Hitz, and N.D. Diem. Recursive algorithm for spectral factorization. IEEE Transactions on Circuits and System 21:742-75,1974

[7] E.J.Anderson, and P.Nash. Linear Progamming in Infinite- Dimensional Spaces.: Theory and Application John Wiley \& Sons, 1987.

[8] E.J.Anderson and A.B. Philpott, editors. Infinite Programming. Springer-Verlag Lecture Notes in Economics and MathematicalSystems, Sept.1984.

[9] Y. Nesterov and A. Nemirovskii, Interior Point Polynomial Methods in Convex Programming. Philadelphia, Philadelphia: SIAM, 1994, vol. 13, Studies in AppliedMathematics.

[10] A. S. Nemirovski and M. J. Todd, "Interior-point methods for optimization," Acta Numerica, vol. 17, pp. 191-234, May 2008. 